ABCDDV/1090

$A B C D$ Arq Bras Cir Dig Letter to the Editor 2015;28(1):86

DOI:http://dx.doi.org/10.1590/S0102-67202015000100021

\section{SPLENIC INFERIOR POLE}

\author{
Polo inferior do baço
}

Alcino Lázaro da SILVA

Emeritus Professor of the Federal University of Minas Gerais, Belo Horizonte, MG, Brazil

Financial source: none Correspondence:

Alino Lázaro da Silva

Conflicts of interest: none

Received for publication: 16/01/2014 Accepted for publication: 09/12/2014

$\mathrm{T}$ he spleen, since antiquity, is an organ little known in its duties ${ }^{1,3}$. We conclude that all its physiological role is not known; but what is known, is that it is essential, verified through other means, by post-splenectomy sepsis for example ${ }^{4,5}$.

Anatomical studies developed by Neder, $\mathrm{AM}^{7}$ and Zapala, A. ${ }^{26}$, guided by DiDio and anatomical dissections by Campos Christo' ${ }^{2}$ guided by Resende Alves ${ }^{22}$, led to the conclusion that the spleen has independent territories under vascular standpoint. There are five. The upper pole is supported by the phrenoesophageal membrane and few phrenic vessels and the inferior pole presents the splenicgastric membrane, sectorial pedicle and the spleno-colic ligament. There is, therefore, greater anatomical integration of inferior pole with the visceral abdominal compartment, mainly with left upper quadrant ${ }^{6-8}$.

Young stabbing victim forced Campos.Christo ${ }^{2}$ to have the opportunity to realize the first partial splenectomy (ruled) successfully. The fact spread and today, injured spleen is maintained spleen ${ }^{9-14}$.

We had the opportunity to implant spleen fragments experimentally and all took completely. Petroianu discussed with me, but did not accept the suggestion and decided to investigate the upper pole on excellence studies ${ }^{15-21}$ on its remaining function after ruled splenectomy.

In contrast, thinking, suggested that the inferior pole provides anatomical conditions superior to those of the upper pole ${ }^{23-25}$. We therefore suggested to Prof. Danilo and staff to investigate this idea and proposal, which resulted in various publications.

Why invest in this idea, that is, the lower pole has greater importance in remaining active than the superior pole? It seems puzzling this proposal because, anatomically, the lower pole has as its support the following: splenic pedicle, specific sectorial pedicle, lower gastrosplenic ligament, whole spleno-colic ligament, plentiful in support tissue and has small remnant of splenorenal membrane.

Without making reasoning with sophistry, it seems that it is not unreasonable to expect the lower pole, supported by more ligaments, will do physiological demand more easily and to encourage the formation of a new pedicle, autonomous, to meet the physiological needs of a functioning spleen.

\section{REFERENCES}

1. Balsalobre B, Carbonell-Tatay F. Cellular immunity in splenectomized patients. J Investig Allergol Clin Immunol. 1991 Aug;1(4):235-8.
2. Campos-Christo M. Esplenectomias parciais regradas. O Hospital,1959; 56 (4): 93-8.

3. Crosby WH. An historical sketch of splenic function and splenectomy. Lymphology. 1983 Jun;16(2):52-5.

4. Greco RS, Alvarez FE. Protection against pneumococcal bacteremia by partial splenectomy. Surg Gynecol Obstet. 1981 Jan;152(1):67-

5. Hosea SW, Brown EJ, Hamburger MI, Frank MM. Opsonic requirements for intracellular clearance after splenectomy. $\mathrm{N}$ Engl J Med 1981; 304:245-50.

6. Mayo WJ. Principles underlying surgery of the spleen. JAMA, 1910; 54:14-8.

7. NEDER, A.M.: Estudo anatômico sobre as zonas venosas lienais e sua drenagem no homem (tese de doutoramento). Belo Horizonte (MG) : Faculdade de Medicina da Universidade Federal de Minas Gerais ; 1958

8. Nunes TA, Silva AL. Estudo radiológico da anastomose esplenorenal proximal, através da veia gastro-omental direita, em pacientes portadores de hipertensão porta esquistossomótica. GED. Gastroenterologia e Endoscopia Digestiva, 1995; 14: 257-258

9. Paulo ICAL, Paulo DNS, Lázaro da Silva A, Foletto RM, Colnago GL, Vargas PM. Níveis de lipides plasmáticos em ratos submetidos à esplenectomias total, ligadura simultânea dos vasos esplênicos e a esplenectomia subtotal com preservação do pólo inferior. Rev. Col. Bras., 2005; 32(5): 229-36.

10. Paulo DNS, Lázaro da Silva A, Cintra LC. Bof AM, Santiago DC Ribeiro GB, Esplenectomia subtotal, em cães com preservação do pólo inferior suprido por vasos do ligamento gastroesplênico. Rev. Col. Bras. Cir., 199; 26(3): 147-52.

11. Paulo DNS, Paulo ICAL, Loureiro ER, Fausto EL, Grillo Jr. LSP, Silva AL, Viabilidade do baço de ratos após a ligadura simultânea da artéria e veia esplênica. Rev. Col. Bras. Cir., 205; 32(1): 32-5

12. Paulo MS, Paulo IC, Nunes TA, Silva AL, Cintra LC, Paulo DN. Effect of hyperbaric oxygen therapy in rats with subtotal splenectomy preserving the inferior pole. Acta CIr. Bras., 2010; 26(3); 156-8.

13. Paulo DN, Paulo IC, Kalil M, Vargas PM, Silva AL, Baptista JF, Guerra AJ. Subtotal splenectorhy preserving the lower pole in rats; technical, morphological and functional aspects. Acta Cir. Bras. 2006; 21(5): 321-7.

14. Paulo ICAL, Paulo DNS, Ferrari TA, Azevedo TGV, Silva AL. O pólo inferior do bajDO de ratos e a oxigenoterapia hiperbárica. Rev. Assoc. Med. Bras., 2008, 54(1): 77-81.

15. Petroianu A, Veloso DFM, Costa GR, Alberti LR. Efeitos de operações sobre o baço no lipidograma de ratas. Rev. Assoc. Med. Bras., 2006; 52(1): 56-9.

16. Petroianu A. Esplenectomia subtotal e anastomose esplenorrena proximal para o tratamento da hipertensão portal. Rev. Bras. Cir., 1983; 73(2): 101-4.

17. Petroianu A. Avaliação da função fagocitária em remanescentes de esplenectomia subtotal e autoimpiante autógeno. Rev. Bras, HematoL Hemoter., 2003: 25(1): 25-30.

18. Petroianu A. Esplenectomia subtotal preservando o pólo superior suprido pelos vasos esplenogástricos. Rev. Col. Bras. Cir., 1994, 21(1): $21-6$

19. Petroianu A, Silva KDA. Esplenectomia subtotal para tratamento do hipodesenvolvimento somático e sexual secundário a esplenomegalia. Rev Col. Bras. Cir., 2002; (29)4: 209-12.

20. Petroianu A, Veloso DFM, Costa GR, Alberti LR. Efeitos de operaç̃ões sobre o baço no lipidograma de ratas. Rev Assoc. Med. Bras., 2006; 52(1): 56-9

21. Petroianu A, Resende V, Silva RG, Alberti LR. Avaliação tardia de pacientes submetidos à esplenectomia subtotal: avaliação clínica, laboratorial, imaginológica e funcional com preservação do pólo superior esplênico . Einstein (São Paulo), 2008; 6(3): 247-52.

22. Resende $V$, Petroianu $A$, Subtotal splenectomy for treatment of severe splenic injuries. J. Trauma, 1998; 44(5): 933-5.

23. Saba TM. Physiologyand physiopathology of the reticuloendothelial system. Arch Intern Med. 1970 Dec;126(6):1031-52.

24. Shennib H, Chiu RC, Mulder DS. The effects of splenectomy and splenic implantation on alveolar macrophage function. J Trauma. 1983 Jan;23(1):7-12.

25. Sherman R. Perspectives in management of trauma to the spleen: 1979 presidential address, American Association for the Surgery of Trauma. J Trauma. 1980 Jan;20(1):1-13.

26.Zappalá, A. Estudo anatômico da divisão terminal da artéria lienal: zonas arteriais do baço (tese de livre-docência). Belo Horizonte (MG): Universidade Federal de Minas Gerais, Faculdade de Medicina; 1958 